\title{
State of the art transoral robotic surgery for obstructive sleep apnea-hypopnea syndrome
}

This article was published in the following Dove Press journal:

Robotic Surgery: Research and Reviews

25 May 2016

Number of times this article has been viewed

\section{Mahalakshmi \\ Rangabashyam' \\ Wenjie Huang ${ }^{2}$ \\ Ying $\mathrm{HaO}^{3}$ \\ Hong Juan Han ${ }^{1,4,5}$ \\ Shaun Loh' \\ Song Tar Toh ${ }^{1,2,4,5}$}

'Sleep Apnea Surgery Service, Department of Otolaryngology,

Singapore General Hospital, ${ }^{2}$ Yong

Loo Lin School of Medicine, National

University of Singapore, ${ }^{3} \mathrm{Health}$

Services Research and Biostatistics

Unit, Singapore General Hospital,

${ }^{4}$ Sleep Disorders Unit, Singapore

General Hospital, ${ }^{5}$ Duke-NUS

Graduate School of Medicine,

Singapore
Correspondence: Song Tar Toh

Sleep Apnea Surgery Service,

Department of Otolaryngology,

Singapore General Hospital,

20 College Road, Singapore 169856

Tel +65 632I 4468

Email songtar@gmail.com
Objective: To review the existing literature on the role of transoral robotic surgery (TORS) for tongue base reduction in the management of adult obstructive sleep apnea-hypopnea syndrome (OSAHS).

Methods: We searched PubMed, MEDLINE, and Scopus databases from the first literature report of this surgical technique to July 30, 2015 for studies investigating the use of TORS for tongue base reduction in treating adult OSAHS. Our primary outcome measures were ApneaHypopnea Index (AHI), lowest oxygen saturation (LSAT), Epworth Sleepiness Score (ESS), and the rates of surgical cure $(\mathrm{AHI}<5)$ and success $(50 \%$ reduction in $\mathrm{AHI}$ accompanied by a postoperative $\mathrm{AHI}<20$ ). Our secondary outcome measures were the volume of tissue resected and correlation to AHI, polysomnographic parameters, subjective outcomes, and body mass index. Complications of surgery were also analyzed.

Results: Thirteen articles were critically evaluated for this research. However, only four case series qualified for statistical analysis of postoperative polysomnographic outcomes and six case series for analysis of postoperative complications. They were case series with a total of 451 adult patients. Pooled analysis revealed statistically significant improvements in AHI, LSAT, and ESS after surgery by 26.83 /hour, $5.28 \%$ and -8.03 , respectively. The average rates of surgical cure and success were $23.8 \%$ and $66.7 \%$, respectively. No study reported any deaths or complications related to the use of robotic equipment. The major complication rate was $6.9 \%$, and the minor complication rate was $30.0 \%$. Major complications included major bleeding (2.9\%), severe odynophagia with dehydration (3.3\%), and oropharyngeal stenosis ( $0.7 \%)$. Minor complications included transient bleeding (0.5\%), transient dysphagia (3.8\%), and dysgeusia (6.6\%).

Conclusion: TORS for tongue base reduction, as a component of multilevel surgery, is an effective treatment option for OSAHS with an acceptable morbidity. This conclusion is based on the analysis of the results of multiple case series. Future studies should entail prospective randomized controlled trials with larger sample size for longer follow-up period.

Keywords: transoral robotic surgery, tongue base resection, base of tongue, multilevel surgery, hypopharyngeal collapse, retroglossal airway

\section{Introduction}

Obstructive sleep apnea-hypopnea syndrome (OSAHS) is a complex disease associated with repeated multilevel upper airway collapse during sleep, resulting in oxygen desaturations, arousals, and sleep fragmentation. Untreated OSAHS can lead to increased risk of coronary artery disease, myocardial infarction, stroke, hypertension, cardiac arrhythmias, and neurocognitive deteriorations and can contribute to poor quality of life and increased risk of all-cause mortality. ${ }^{1-4}$ 
The gold standard for treating OSAHS is positive airway pressure (PAP) therapy. However, PAP therapy is often limited by poor compliance and adherence. ${ }^{5,6}$ In patients who rejected PAP therapy, upper airway surgery is an established alternative treatment option.

According to American Association of Sleep Medicine (AASM) 2010 practice guidelines, it is not recommended to treat moderate-to-severe OSAHS with isolated uvulopalatopharyngoplasty (UPPP) procedure or palatopharyngeal reconstruction, since it is ineffective in normalizing ApneaHypopnea Index (AHI). ${ }^{7}$ Caples et al reported that isolated UPPP reduces the AHI by $33 \%$, and the mean postoperative AHI can remain as high as $30.0 /$ hour. $^{8}$ OSAHS is a disease characterized by multilevel airway collapse. It is therefore logical that multilevel surgery addressing the different sites of obstruction may yield improved outcomes. ${ }^{9}$

The advent of drug-induced sleep endoscopy in the past decade has demonstrated that the base of tongue (BOT) and supraglottic regions are key sites of hypopharyngeal collapse in $>38 \%-56 \%$ of patients. ${ }^{10,11}$ The possible factors include hypertrophied lymphoid tissue, altered genioglossus muscle tone, increased fat deposition at BOT, and retrognathia. ${ }^{12-15}$ The aim of hypopharyngeal OSAHS surgery is to widen the retroglossal airway space.

The hypopharyngeal tongue-related interventions include tongue reduction procedures, such as radiofrequency-BOT (RF-BOT), midline glossectomy, lingualplasty, and submucosal minimally invasive lingual excision (SMILE), and procedures that can increase tension and widen posterior airway space at the tongue base, such as genial tubercle advancement and hyoid suspension. An evidence-based review reported that although many procedures have evolved to enlarge the retroglossal airway, consistency in results was lacking. ${ }^{16}$ Additionally, more aggressive tongue reduction procedures were limited by access and anatomical constraints for safe resection, with the risk of injury to lingual artery and hypoglossal nerve.

Transoral robotic surgery (TORS) was initially developed in 2006 to treat upper aerodigestive tract neoplasms by O'Malley et al. ${ }^{17}$ In 2010, Vicini et al utilized TORS for volumetric tongue reduction to widen the retroglossal airway in the management of OSAHS. ${ }^{18}$ The promising results of his case series led to the novel application of TORS for tongue base reduction in the surgical management of OSAHS, as a component of multilevel surgery, for patients who have failed or rejected PAP therapy and medical management.

Several case series have been published on the effectiveness and safety profile of TORS for tongue base reduction to date. However, there are no reviews focusing purely on the outcomes of TORS for tongue base reduction in OSAHS as a key component of multilevel surgery. The aim of this research was to analyze existing relevant literature and evaluate the effectiveness and complications of TORS for tongue base reduction in the treatment of OSAHS.

\section{Materials and methods Search strategy}

A PubMed, MEDLINE, and Scopus search was performed from the inception of this surgical technique to July 30 , 2015. The primary keywords used included "transoral robotic surgery" and "sleep apnea syndrome". Other keywords were "obstructive sleep apnea", obstructive sleep apnea hypopnea syndrome", "robotic surgery", "tongue base", "base of tongue", "retro-lingual collapse", "supraglottoplasty", and "hypopharyngeal collapse". References of all relevant articles and relevant nonelectronic literature were handsearched to identify additional studies. Hand-searching identified four more studies. The abstracts of all articles were screened, and the full text of possibly relevant articles was reviewed.

\section{Inclusion criteria}

The inclusion criteria are shown in Figure 1 and are as follows:

1. Adult OSAHS patients 18 years of age and above who underwent TORS.

2. Articles that reported preoperative and postoperative data for at least one primary outcome and/or surgical complications.

\section{Exclusion criteria}

The exclusion criteria are shown in Figure 1 and are as follows:

1. Pediatric population

2. Studies not available in English

3. Manuscripts discussing TORS but not in the context of OSAHS treatment (eg, malignancies)

4. If the indication of OSAHS for performing TORS was in less than $20 \%$ of the patient population.

5. Cadaveric studies and those focused on anatomy and surgical technique with no mention of postoperative outcomes

6. Preliminary studies ${ }^{18,19,20}$ whose findings had been nested in larger studies that were subsequently published ${ }^{21,22,23}$

7. Sample size smaller than five patients ${ }^{24}$

\section{Data extraction}

Data from manuscripts were analyzed and evaluated for inclusion independently by two reviewers (MR and WH). 


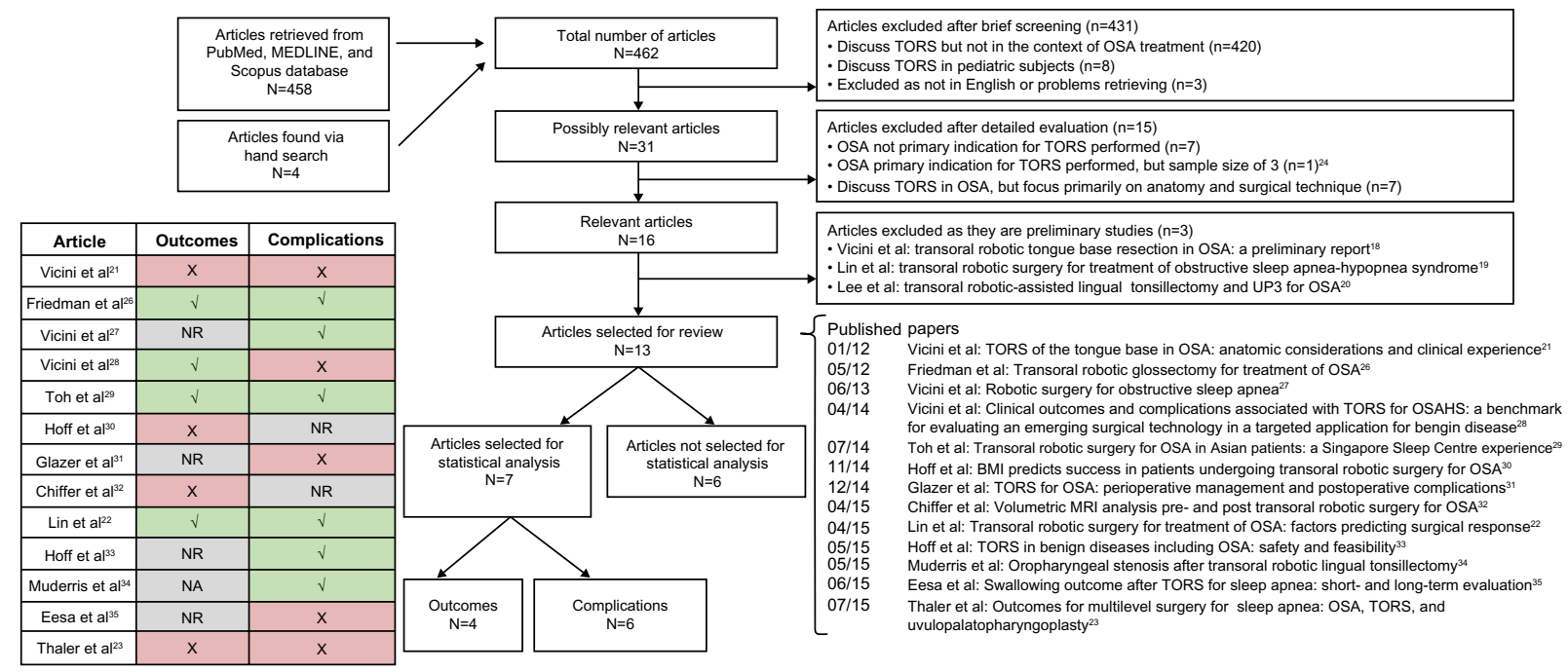

Figure I Literature search strategy, study inclusion and exclusion criteria, and selection for statistical analysis.

Abbreviations: NR, not reported; NA, not applicable; TORS, transoral robotic surgery; OSA, obstructive sleep apnea; OSAHS, obstructive sleep apnea-hypopnea syndrome; $\mathrm{MRI}$, magnetic resonance imaging.

Primary outcome measures included preoperative and postoperative AHI, lowest oxygen saturation (LSAT), Epworth Sleepiness Score (ESS), and the rates of surgical cure and surgical success.

Secondary outcome measures consisted of volume of tissue resected and correlation to AHI, other polysomnographic (PSG) parameters, subjective outcomes, and body mass index (BMI). Complications of surgery and study details such as sample size, patient demographics, and concomitant procedures performed were also collected.

\section{Study quality assessment}

A quality appraisal tool from National Institute for Health Clinical Excellence (NICE) to assess methodological quality of the included studies was utilized. ${ }^{25}$

\section{Statistical analysis}

Studies that had overlapping recruitment periods from the same author were carefully analyzed to minimize bias in the pooled results (Figure 1).

The meta-analysis of the results of AHI, LSAT, ESS, and BMI was done using the pre- and posttreatment values with Review Manager (RevMan) Version 5.3 (The Nordic Cochrane Centre, The Cochrane Collaboration, 2014, Copenhagen, Denmark).

Only studies that provided mean and standard deviation of the mentioned outcomes were included. The fixed effects model and the random effects model were used in this study depending on the presence of statistical heterogeneity, which was assessed using the $I^{2}$ statistic. Where this heterogeneity test yielded a $P$-value $>0.05$ (for the outcomes of AHI and BMI), the fixed effects model was used. Where it yielded a $P$-value $<0.05$ (for LSAT and ESS), the random effects model was used to better reflect variation between the different studies. We calculated the effect sizes using mean differences (MD). Standardization of the MDs was not required as all authors measured the outcomes using the same metric. If the $95 \%$ confidence interval (CI) of MD did not cut 0 , the MD was considered statistically significant.

Finally, visual inspection of the funnel plots was also performed for all outcomes (data not presented), which revealed no publication bias.

\section{Results}

We identified 462 potentially relevant articles from our electronic and handsearching (Figure 1), and 431 articles were excluded after screening their abstracts. Majority of the articles discussed the utility of TORS only in malignancies and/or other benign conditions.

The remaining 31 articles were retrieved for full-text evaluation, and an additional 15 articles were excluded: seven presented pooled data, with OSAHS being the indication for TORS in $<20 \%$ of cases; one had a small sample size; and even focused primarily on surgical techniques. Of the 16 articles that remained, three articles were further excluded since they were preliminary studies. ${ }^{18-20}$ Their data had been nested in larger studies that were subsequently published. ${ }^{21-23}$

Thirteen articles were finally reviewed (Figure 1). ${ }^{21-23,26-35}$ Studies that had overlapping recruitment periods from the 
same author were carefully analyzed to minimize bias in the pooled results. Consequently, one article ${ }^{28}$ was used for the analysis of outcomes only, three for complications only, ${ }^{27,33-34}$ and three for both outcomes and complications (Table 1, Figure 1). 22,26,29

\section{Study quality assessment}

All of the studies were case series, with the exception of one case study. ${ }^{34}$ Most of the articles satisfied four to five of the eight items on the NICE Quality Assessment checklist (Table 1). With the exception of two multicenter studies by Vicini et al and Hoff et al, the remainder of the studies were single-institution reports. ${ }^{28,33}$

\section{Inclusion criteria}

Inclusion and exclusion criteria varied across studies. However, patients were generally included if 1 ) they were above 18 years of age and had symptomatic or moderate-to-severe OSAHS, as diagnosed by PSG, 2) rejected or failed conservative management such as PAP therapy, 3) had evidence of retroglossal obstruction during drug-induced sleep endoscopy, and 4) had no contraindication to surgery.

\section{Exclusion criteria}

Patients were excluded in the studies if they did not undergo a postoperative PSG or if they had undergone previous surgical treatment for OSAHS. ${ }^{26,29}$

\section{Demographics}

Thirteen studies were reviewed..$^{21-23,26-35}$ Excluding potential duplication of data yielded a total of 451 distinct patients, most of whom were male (76.1\%) with a mean age of 49.8 years. ${ }^{22,26,29,33-35}$ The studies were mostly held in the USA and Europe, with only one study being conducted in the Asian population. ${ }^{29}$

\section{Surgical technique}

Robotic tongue base reduction was performed as a component of multilevel surgery in conjunction with other upper airway modification surgery (nasal, palatal, and pharyngeal) in the same setting (Table 1). All series adopted the inverted pyramid technique mentioned by Vicini et al, with the exception of Friedman et al who adopted the triangular resection technique. $^{18,26}$

\section{Robotic time}

Robotic setup time and robotic operative time were reported by three case series. ${ }^{22,28,29}$ The average robotic setup time was 24.8 minutes, and average robotic operative time was 30.8 minutes (Table 2). ${ }^{22,28,29}$ Vicini et al and Toh et al reported that robotic setup and robotic operative time declined with increasing experience..$^{21,29}$

\section{Duration of stay}

The average duration of stay was 3.2 days (Table 3 ), ${ }^{22,26,29,33-35}$ ranging from $1.8 \pm 1.3$ to $9.5 \pm 2.9$ days.$^{21,33}$ The main factors influencing the length of stay included the presence of tracheostomy, degree of pain, and tolerance to oral feeds.

\section{Primary outcomes \\ Apnea-hypopnea index}

Four studies comprising 329 patients reported a reduction in AHI from 44.1 /hour to 17.9 /hour by $59.4 \%$ (Table 4 ; Figure 2A). ${ }^{22,26,28,29}$ Pooled analysis of these same studies revealed a statistically significant improvement of -26.83 (95\% CI, -29.89 to -23.76) (Figure 3A).

Given the inconsistency in terminology used, we elected to define "surgical cure" as a "postoperative AHI of $<5$ " and "surgical success" as a " $50 \%$ reduction in AHI following surgery accompanied by a postoperative AHI of $<20$ ", as data are most available for these two measures. Based on this definition, the average rates of surgical cure and success are $23.8 \%$ and $66.7 \%$, respectively (Table 4 ). The true surgical success rate is probably slightly upward of this value, as two authors presented data using stricter benchmarks of AHI and ESS. 22,28

\section{Lowest oxygen saturation}

Four studies totaling 329 patients reported a $6.3 \%$ improvement in LSAT from $79.0 \%$ to $84.0 \%$ postoperatively (Table 4 ; Figure 2B). 22,26,28,29 Pooled analysis of these same studies revealed a statistically significant improvement of $5.28 \%$ (95\% CI, 2.44-8.11) (Figure 3B).

\section{Epworth Sleepiness Score}

Four studies totaling 329 patients reported a 55.2\% improvement in ESS from 12.8 to 5.7 postoperatively (Table 4; Figure 2C). 22,26,28,29 Pooled analysis of these same studies revealed a statistically significant improvement of -8.03 (95\% CI, -9.81 to -6.25 ) (Figure 3C).

\section{Secondary outcomes}

\section{Volume of tissue resected and correlation to results}

Six studies reported the volume of tissue resected, with resection amount varying widely across them. ${ }^{22,26,28-31}$ Resected BOT tissue is typically measured in milliliters $(\mathrm{mL})$ through 


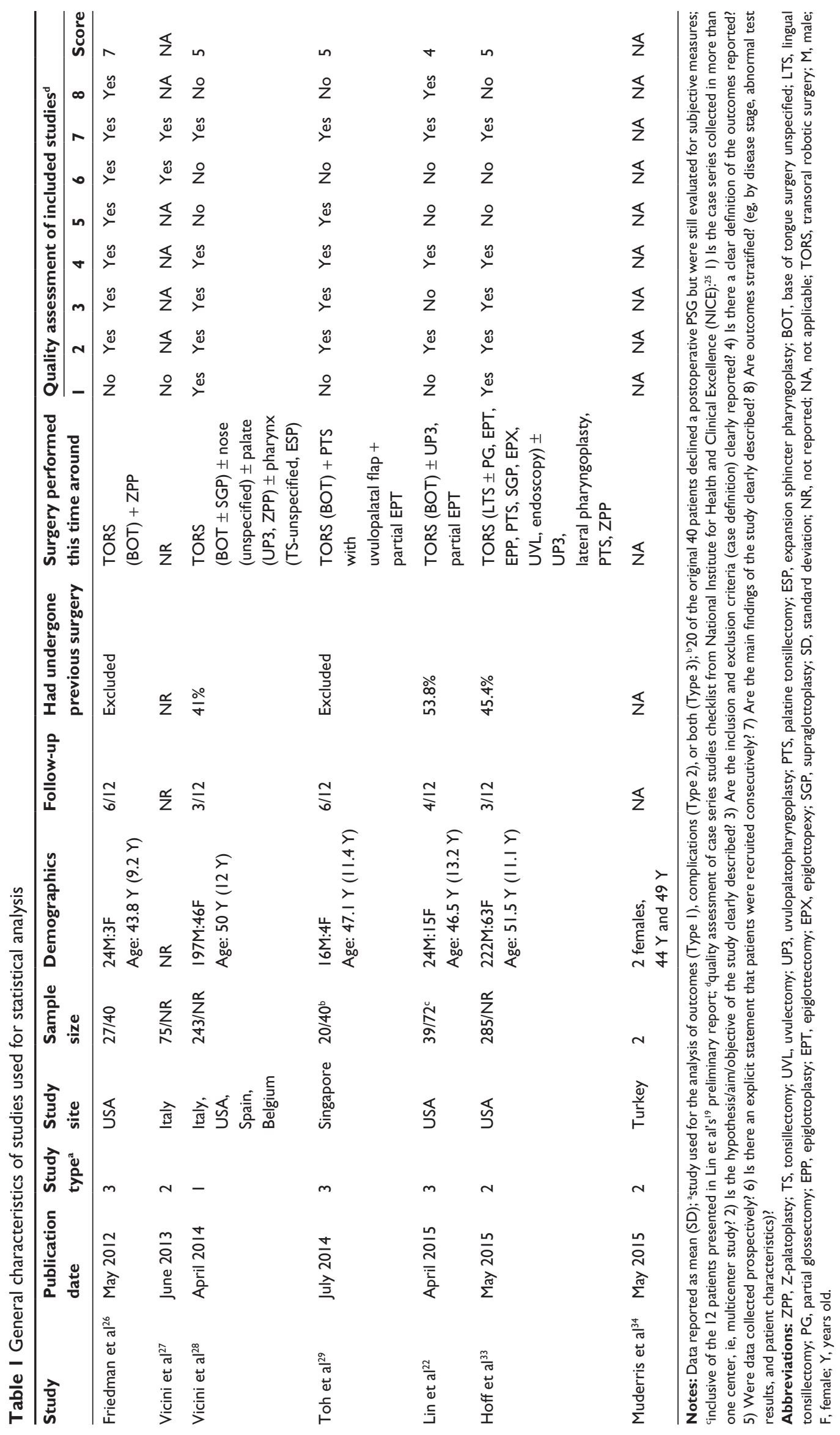




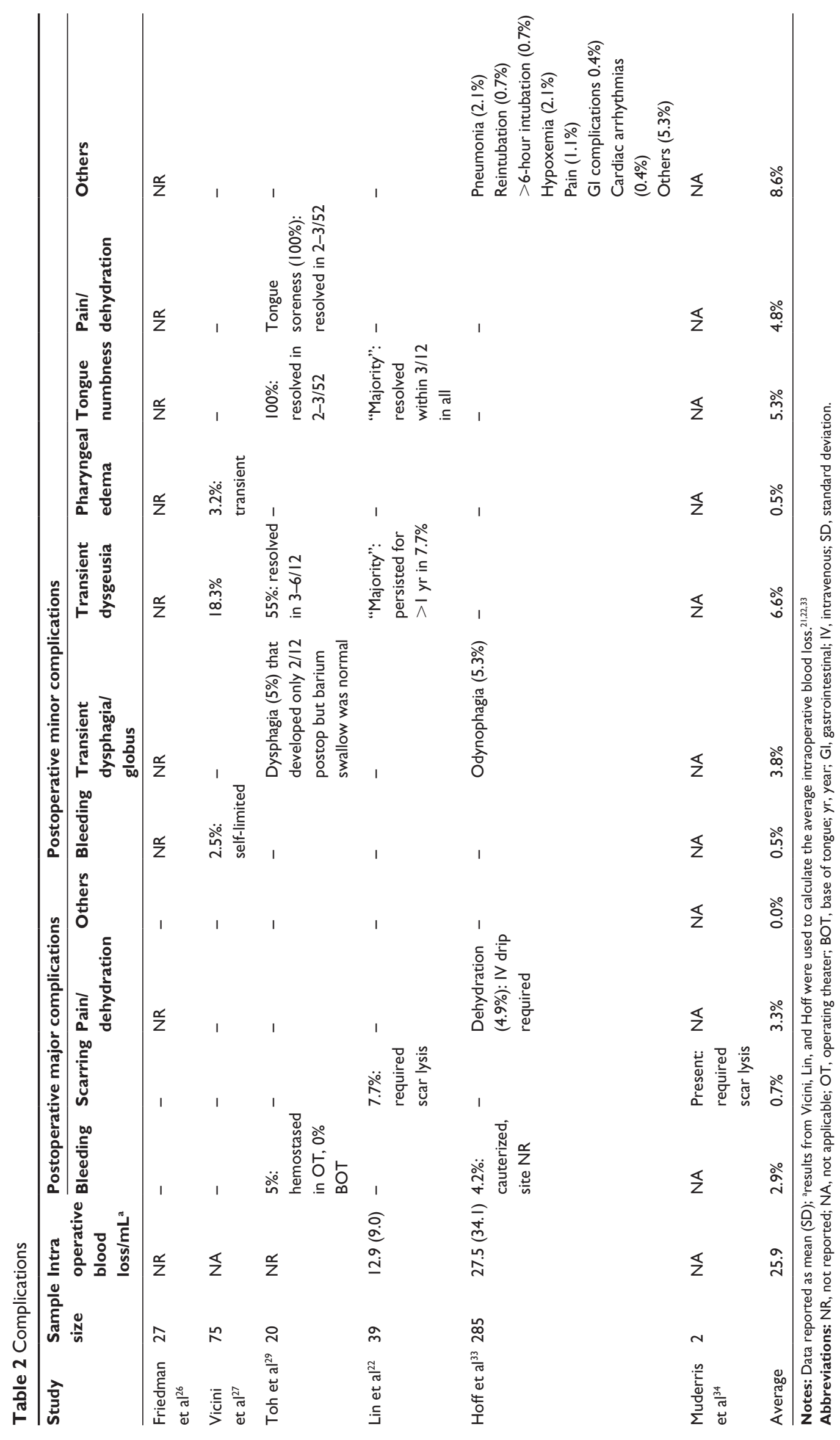


A

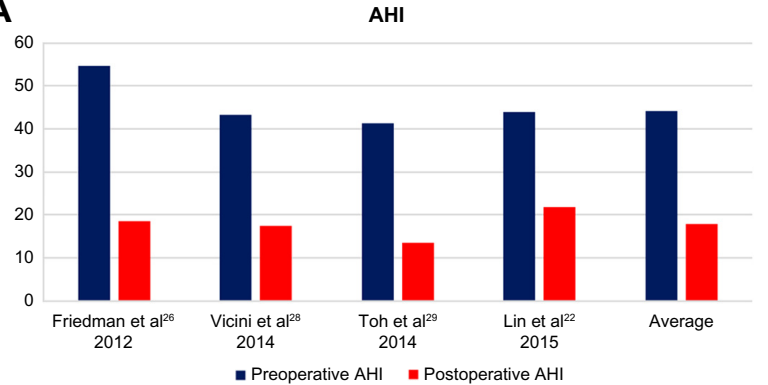

C

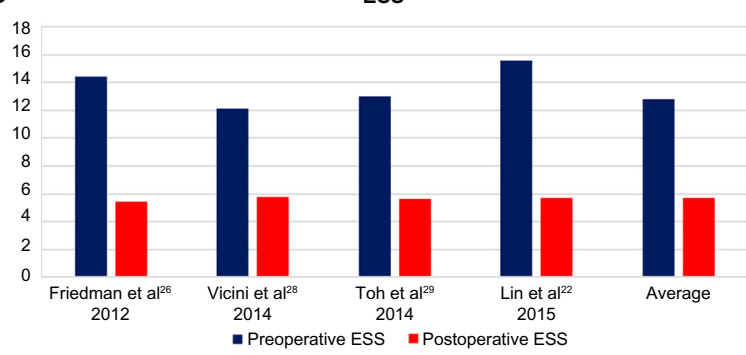

B

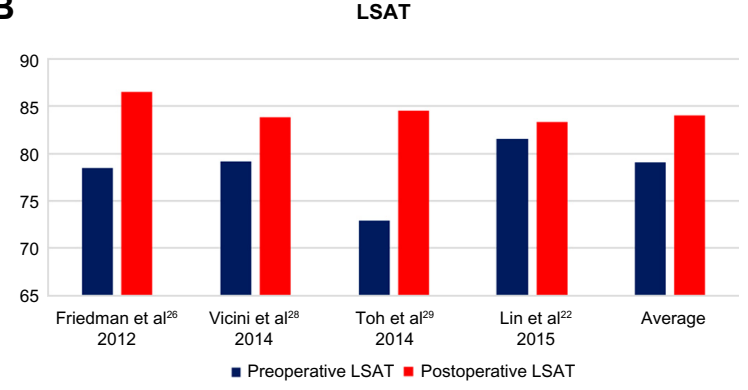

D

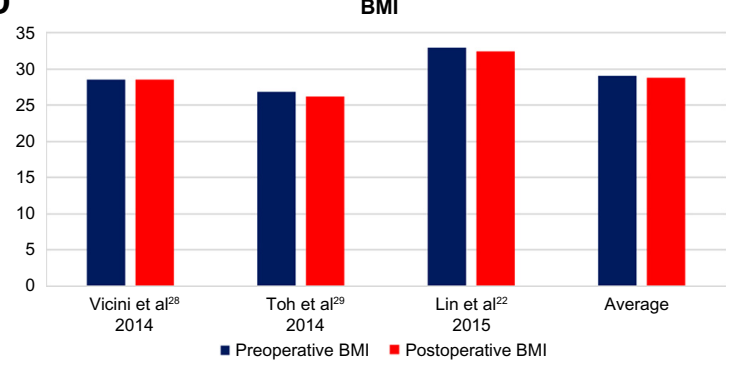

Figure 2 Graphical representation of (A) AHI, (B) LSAT, (C) ESS, and (D) BMI.

Abbreviations: AHI, Apnea-Hypopnea Index; LSAT, lowest oxygen saturation; ESS, Epworth Sleepiness Score; BMI, body mass index.

the displacement technique by immersing the tissue in a half-filled calibrated syringe or cup and reading the volume of fluid displaced. ${ }^{21-23,27-35}$ The volume of tissue resected was in the range of 9-10 mL. ${ }^{28,29}$ However, one study reported resection volume as high as $22.2 \mathrm{~mL} .^{22}$ The average volume of tissue resected, based on the analysis of results from four studies, was $11.0 \mathrm{~mL}$ (Table 4). ${ }^{22,26,28,29}$ Friedman et al, however, used the metric of weight in grams $(\mathrm{g})$ and resected an average of $2.28 \mathrm{~g} .{ }^{26}$ Eesa et al reported that a minimum of 7 $\mathrm{mL}$ resection volume was required to relieve the symptoms of BOT obstruction. ${ }^{35}$ Hoff et al found that a lower resection volume (6.07 vs $8.24 \mathrm{~mL}$ ) was significantly associated with poorer outcomes. ${ }^{30}$

\section{Other polysomnographic parameters}

Toh et al and Thaler et al highlighted crucial PSG parameters apart from AHI that improved statistically significantly following surgery. ${ }^{23,29}$ These parameters were improvement in sleep architecture and the amount of N3 and REM sleep, reductions in the amount of N1 sleep, respiratory disturbance index, oxygen desaturation index, and hypoxic time..$^{23,29}$ Total sleep time with $<90 \%$ oxygen saturation (hypoxic time) decreased from $18.2 \%$ (20 minutes) to $3.4 \%$ (6.8 minutes) in one study and in the second study from $16.9 \%$ to $7.2 \% .^{23,29}$

\section{Subjective outcomes measures}

A visual analog scale was also employed by various authors to evaluate subjective outcomes according to patients and bed partner's satisfaction. ${ }^{21,29}$ Friedman et al and Toh et al also noted that snoring intensity significantly improved postoperatively. ${ }^{26,29}$ Their respective visual analog scale scores were $9.1 \pm 1.0$ and $9.5 \pm 0.6$ preoperatively and $2.3 \pm 2.9$ and $2.5 \pm 1.8$ postoperatively. ${ }^{26,29}$

\section{Body mass index}

Three studies totaling 302 patients reported a $0.5 \%$ drop in BMI from 29.0 to 28.8 postoperatively (Table 4, Figure 2D). ${ }^{22,28,29}$ Pooled analysis of these same studies revealed that the drop of -0.11 in BMI was not statistically significant (95\% CI, -0.75 to 0.54 ) (Figure 3D).

\section{Complications}

None of the studies reported any deaths or complications related to airway or use of robotic equipment.

\section{Intraoperative}

The volume of blood lost intraoperatively ranged from 12.9 to $27.7 \mathrm{~mL}$ with an average of $25.9 \mathrm{~mL}$ (Table 2). ${ }^{21,22,33} \mathrm{Vicini}$ et al also reported an intraoperative bleeding rate of $0.4 \%{ }^{28}$

\section{Postoperative}

We classified postoperative complications as major and minor (Table 2), depending on the need for surgical exploration and readmission.

Major complications included 1) bleeding necessitating surgical exploration $(2.9 \%), 2)$ oropharyngeal stenosis requiring scar lysis $(0.7 \%)$, and 3 ) poorly controlled pain 


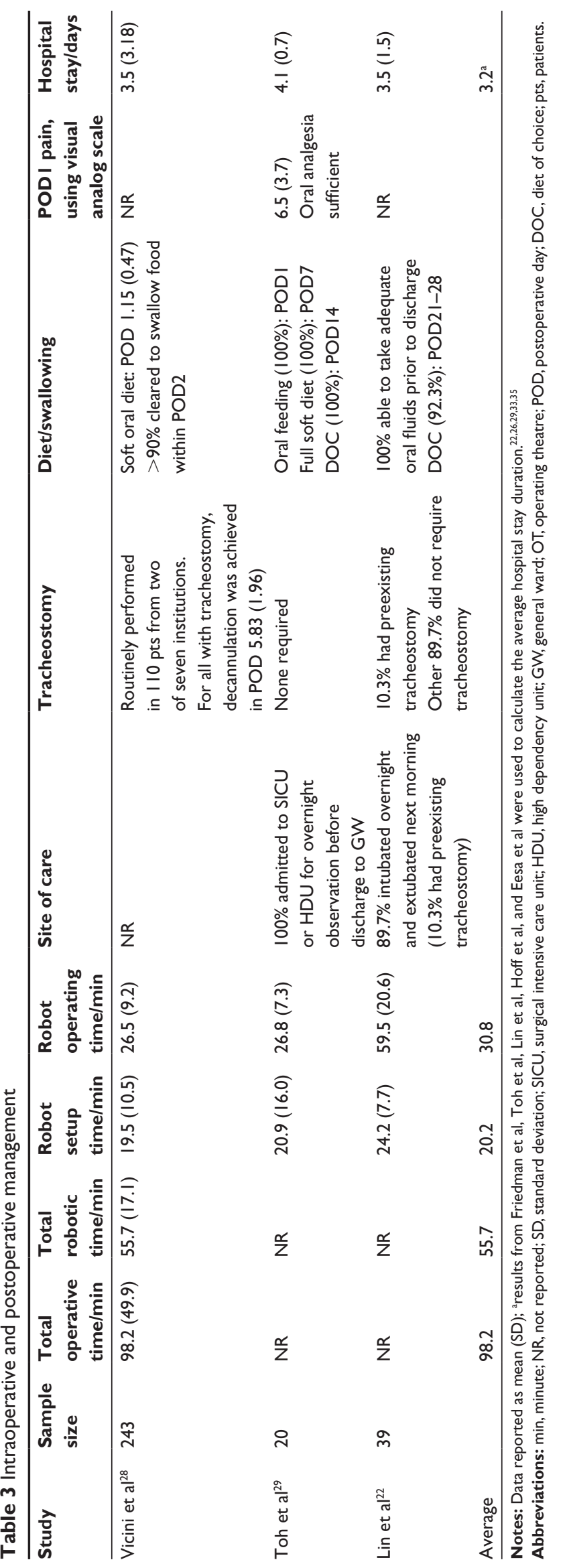

with dehydration necessitating intravenous replacement (3.3\%) (Table 2).

Minor complications included 1) bleeding that could be controlled by the bedside, 2) transient dysphagia, 3) transient dysgeusia, 4) pharyngeal edema, 5) tongue numbness, 6) minor pain and dehydration, and 7) others. All of these complications generally occurred at a low rate of $<5 \%$ (Table 2 ), with the exception of dysgeusia (6.6\%).

Multiple complications occurring in the same patient were double counted if they were independent events. The major complication rate was $6.9 \%$, and the minor complication rate was $30.0 \%$. The minor complication rate is likely to be underestimated as the use of descriptors like "many", "most", "majority" (Table 2) by various authors cannot be translated into quantifiable terms and are thus not factored into calculations..$^{22,31,35}$ Reassuringly though, these descriptors relate to dysgeusia and tongue numbness, which tend to resolve with time.

\section{Discussion}

TORS has become an established option to treat tongue base obstruction in the surgical management of OSAHS as a component of multilevel surgery. Currently, close to 500 patients from various tertiary care centers all over the world have been treated for OSAHS with TORS. Our review is the first to focus primarily on TORS and its role in the management of OSAHS.

This review comprises 13 studies encompassing 451 patients, with a minimum period of 3 months and a maximum period of 20 months follow-up. Majority of the patients were male $(76.1 \%)$ with an average age of 49.8 years.

\section{Primary outcomes}

The efficacy of TORS was found to be consistent across three salient parameters: AHI, LSAT, and ESS. BMI was monitored since reduction in weight could potentially confound these outcomes.

\section{Postoperative AHI, LSAT, and ESS improved significantly}

Our results revealed that across four studies comprising 329 patients, AHI decreased by $59.4 \%$ from 44.1 to 17.9 postoperatively. ${ }^{22,26,28,29}$ This was greater than $33 \%$ reduction with isolated UPPP reported by Caples et al. ${ }^{8}$ However, this comparison to highlight that isolated palatal surgery may be inadequate and multilevel surgery addressing the palate, nasal, and retroglossal airways likely to yield improved outcomes since OSAHS is a complex condition 
A

\begin{tabular}{|c|c|c|c|c|c|c|c|c|}
\hline \multirow[b]{2}{*}{ Study or subgroup } & \multicolumn{3}{|c|}{ Postoperative } & \multicolumn{3}{|c|}{ Preoperative } & \multirow[b]{2}{*}{ Weight } & \multirow{2}{*}{$\begin{array}{l}\text { Mean difference } \\
\text { IV, fixed, } 95 \% \mathrm{Cl}\end{array}$} \\
\hline & Mean & SD & Total & Mean & SD & Total & & \\
\hline$\overline{\text { Friedman et al }}{ }^{26}$ & 18.6 & 9.1 & 27 & 54.6 & 21.8 & 27 & $11.8 \%$ & $-36.00(-44.91,-27.09)$ \\
\hline Vicini et $\mathrm{al}^{28}$ & 17.5 & 16.5 & 243 & 43.2 & 22.6 & 243 & $75.9 \%$ & $-25.70(-29.22,-22.18)$ \\
\hline Toh et $\mathrm{al}^{29}$ & 13.5 & 17.1 & 20 & 41.3 & 22.1 & 20 & $6.3 \%$ & $-27.80(-40.05,-15.55)$ \\
\hline Lin et al ${ }^{22}$ & 21.9 & 23.5 & 39 & 43.9 & 32.3 & 39 & $6.0 \%$ & $-22.00(-34.54,-9.46)$ \\
\hline Total $(95 \% \mathrm{Cl})$ & & & 329 & & & 329 & $100 \%$ & $-26.83(-29.89,-23.76)$ \\
\hline
\end{tabular}

Heterogeneity: $\chi^{2}=5.06, d f=3(P=0.17) ; P^{2}=41 \%$

Test for overall effect: $Z=17.15(P<0.00001)$

B

LSAT

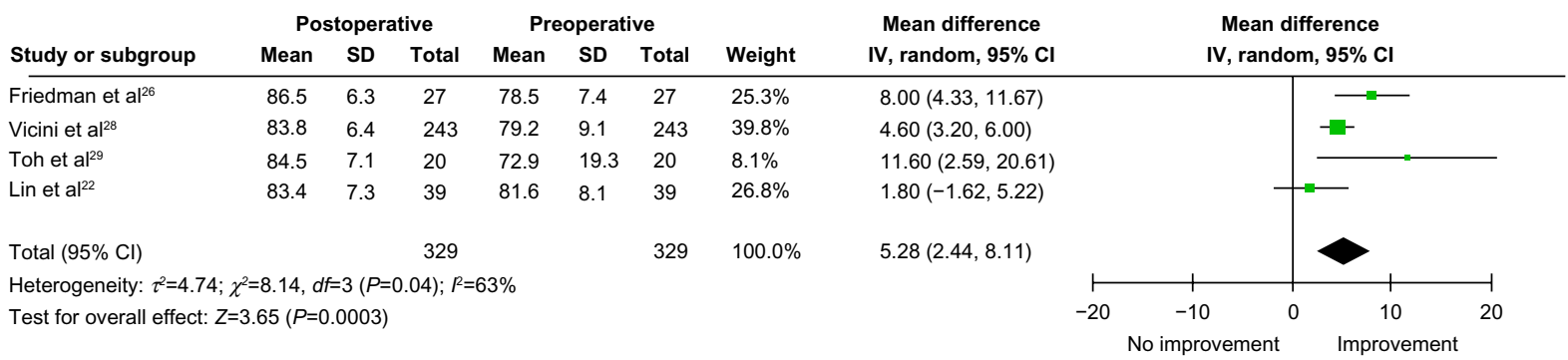

C

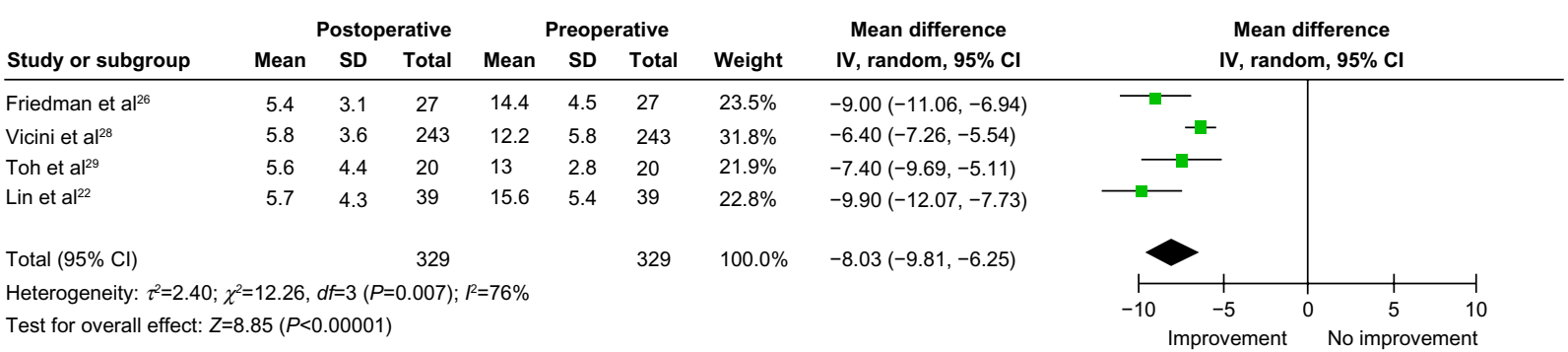

D

BMI

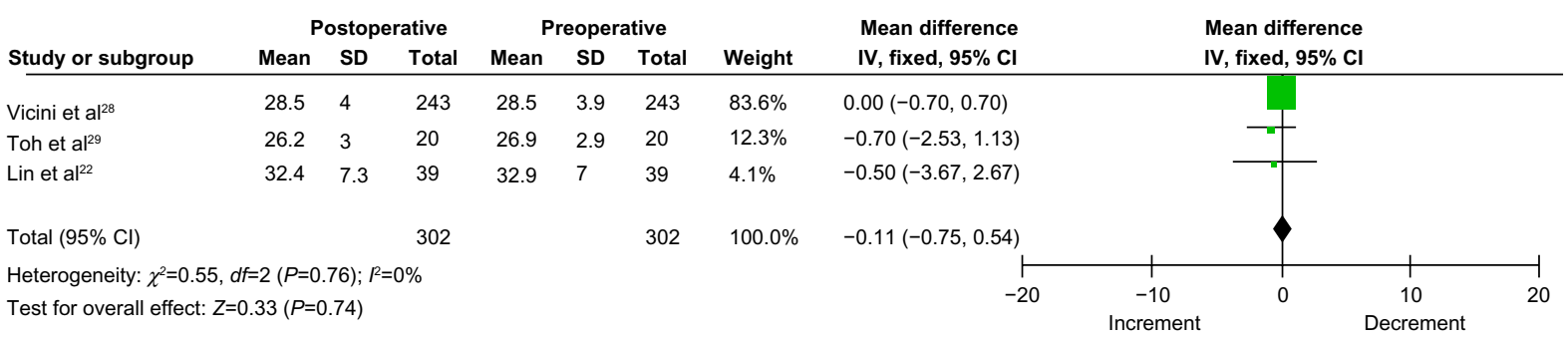

Figure 3 Pooled analysis of (A) AHI, (B) LSAT, (C) ESS, and (D) BMI.

Abbreviations: AHI, Apnea-Hypopnea Index; LSAT, lowest oxygen saturation; ESS, Epworth Sleepiness Score; BMI, body mass index; SD, standard deviation; IV, independent variable; $\mathrm{Cl}$, confidence interval; $\mathrm{df}$, degrees of freedom.

involving multiple sites of obstruction. ${ }^{9}$ The nontreatment of retroglossal narrowing and hypopharyngeal collapse could, therefore, have possibly contributed to the reduced outcomes observed in isolated palatal surgery. Based on our definition, the average rates of surgical cure and success were $23.8 \%$ and $66.7 \%$, respectively. The true surgical success rate is probably slightly upward of this value, as two authors presented data using stricter benchmarks. ${ }^{22,28}$ In four studies totaling
329 patients, LSAT and ESS were assessed. ${ }^{22,26,28,29}$ LSAT increased by $6.3 \%$ from $79.0 \%$ to $84.0 \%$. The ESS is a robust subjective parameter and was found to improve from 12.8 to 5.7 on average, representing a $55.2 \%$ increment.

\section{Surgical cure, success, response, and failure}

There was no consistent definition used across studies, with terms such as surgical "cure", "success", "response", 


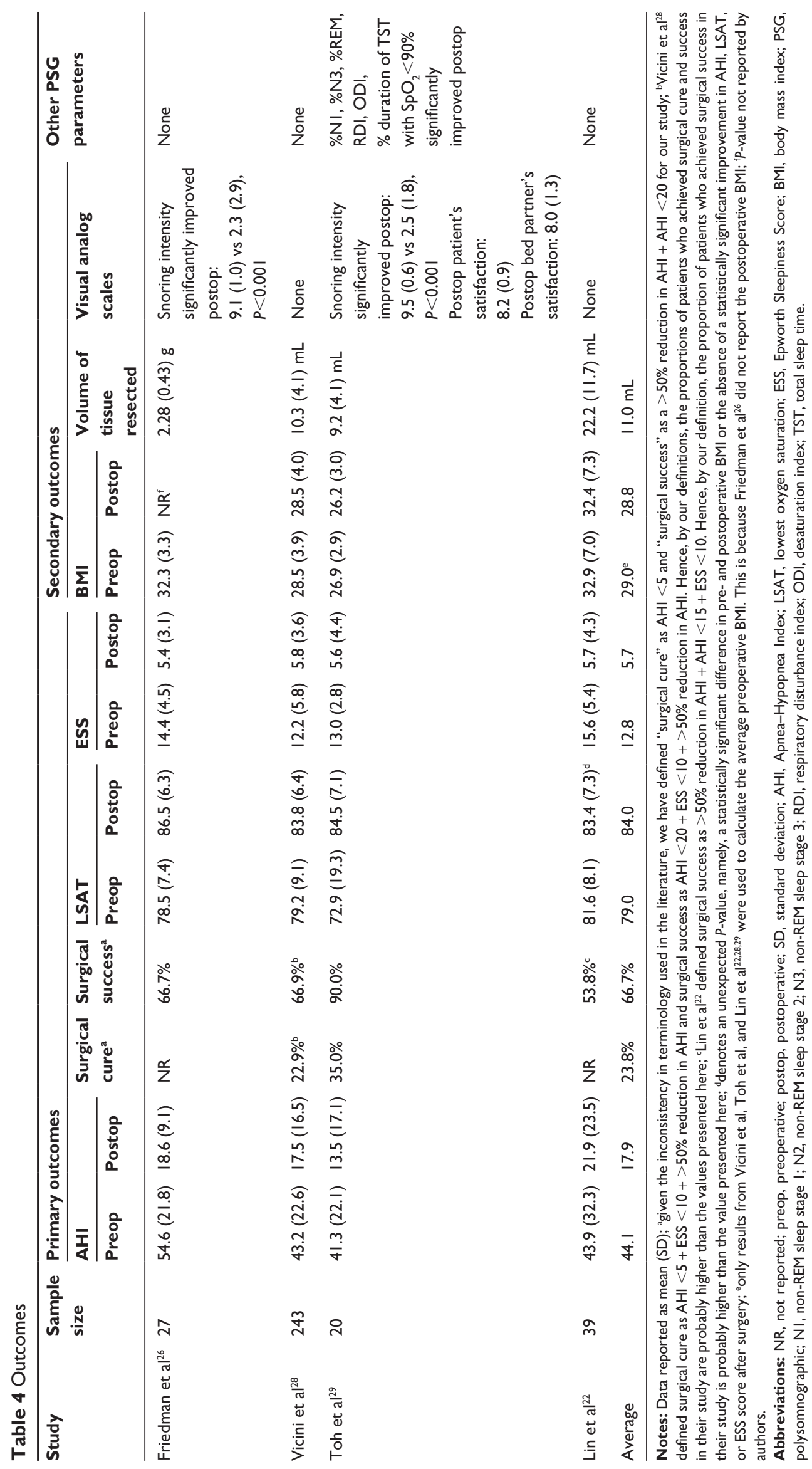


and "failure" being defined differently by most authors. Toh et $\mathrm{al}^{29}$ and Hoff et $\mathrm{al}^{30}$ defined true surgical cure as a postoperative AHI of $<5$, achieving a cure rate of $35.0 \%$ and $14.0 \%$, respectively. Surgical success was commonly defined by a few papers when there was a $>50 \%$ reduction in AHI accompanied by a postoperative AHI of $<20 .{ }^{23,29,30,32}$ However, Friedman et al defined the same as surgical cure. ${ }^{26}$ Two studies had strict guidelines with the inclusion of ESS and AHI. ${ }^{22,28}$ Lin et al categorized his patients as "responders" those who achieved $>50 \%$ reduction from preoperative AHI, a final AHI of $<15$, and ESS $\leq 9$; this was based on Medicare and Medicaid where PAP is not required if $\mathrm{AHI}<15{ }^{22}$ Vicini et al, however, regarded "surgical cure" as a combination of AHI $<5$, ESS $<10$, and a $>50 \%$ reduction in AHI. ${ }^{28}$ They were also the only group to stratify results clearly and define surgical failure clearly as a combination of $\mathrm{AHI}>20$, ESS any value, and $<50 \%$ reduction from preoperative $\mathrm{AHI}{ }^{28}$

\section{TORS as a component of multilevel surgery}

It would be ideal to judge the true merit of TORS for tongue base reduction when the procedure is performed in isolation. However, deriving conclusions of multilevel surgery that includes TORS cannot be regarded as a limitation, since patients with severe OSAHS tend to have multilevel collapse and appropriate management should treat all the sites involved. ${ }^{9}$ This review predominantly comprises summarized outcomes of multilevel surgery with TORS. Follow-up for the majority of patients was $<1$ year, and hence future studies with extended follow-up period may validate results in the long run.

\section{Isolated TORS for tongue base reduction}

In an earlier study published by Lin et al, the authors looked at isolated TORS for tongue base obstruction. ${ }^{19}$ The patients underwent baseline preoperative PSG since a majority of patients had previously undergone other upper airway procedures. They concluded that there was a statistically significant improvement in AHI of $56.2 \% \pm 28.3 \%$ from $43.9 \pm 41.1$ to $17.6 \pm 16.2(P=0.007)$ and $53 \%$ improvement of ESS from $13.7 \pm 5.2$ to $6.7 \pm 4.5(P<0.001)$, but not LSAT $83.3 \pm 5.5$ to $84.0 \pm 6.4(P=0.680) .{ }^{19}$ They also reported that despite undergoing only BOT resection, the postoperative AHI and ESS achieved were similar to other studies where patients underwent multiple procedures. However, this study only had a sample size of 12 patients and the focus was tongue base obstruction that was not treated previously. ${ }^{19} \mathrm{In}$ our current review, only $13.7 \%$ (data not shown) of patients across all studies had isolated BOT resection using TORS. It is not known what the exact outcome for these patients were, since the reported results of individual studies were not always stratified.

\section{Prognostic factors in TORS}

The analysis of prognostic factors and what tilts the balance in favor of cure, failure, and improvement continue to remain challenging in sleep surgery. However, factors elucidated that may influence surgical outcomes in TORS include the volume of tissue resected, BMI, AHI, the site and type of upper airway collapse, and the presence of prior airway surgery.

\section{Volume of tissue resected}

It is logical to assume that the greater the resection volume, the better the response rate. Six studies reported the amount of tissue resected..$^{22,26,28-31}$ Majority of the studies measured resection volume in milliliters $(\mathrm{mL})$ and reported a volume $\geq 7 \mathrm{~mL} \cdot{ }^{22,28-30}$ Eesa et al recommended resecting at least $7 \mathrm{~mL}$ to relieve tongue base obstruction. ${ }^{35}$ Hoff et al reported that there may be a positive correlation between the volume of BOT tissue resected and surgical success and that the group with poorer outcomes had lower volumes resected (6.07 vs $8.24 \mathrm{~mL}) .{ }^{30}$ The study of Friedman et al was the only study to use the metric of weight in grams $(\mathrm{g})$, resecting an average of $2.28 \pm 0.43 \mathrm{~g} .{ }^{26}$ This is estimated to be $2.28 \mathrm{~mL}$ using a conversion of $1.00 \mathrm{~g} / \mathrm{mL}$ based on the density of muscle and fat. ${ }^{26}$ The authors concluded that there was no significant correlation between the weight of lingual tissue excised and degree of improvement in AHI, LSAT, or ESS. ${ }^{26}$ This finding may probably be due to the smaller volume of tissue resected in their study.

\section{Body mass index}

Preoperative

Hoff et al found that patients with a lower preoperative BMI had higher chance of surgical success: the proportion of patients who had surgical success with BMIs of $<30$ and $<25$ were $56.5 \%$ and $78.3 \%$, respectively. ${ }^{30}$ Lin et al found that patients with a preoperative BMI $<30$ had an excellent surgical response (AHI $<15$ and ESS $\leq 9)$ rate of $88.2 \%$ and those with BMI $>40$ had a poor response of $16.7 \% .^{22}$

It is well established that an elevated BMI is associated with increased tongue volume, macroglossia, and airway collapsibility through various mechanisms. ${ }^{36,37}$ It may, therefore, be prudent to consider tongue reduction over tensioning procedures due to the increased tongue volume in the presence of raised BMI. 
Asian men have been known to have more severe OSAHS, despite a low BMI on account of skeletal framework. ${ }^{38}$ Toh et al published his data involving primarily Asian population and reported cure in seven out of 20 patients (35\%), traditional success in another eleven patients (55\%), and cumulative surgical success in 18 patients $(90 \%)$, despite the skeletal disadvantage. ${ }^{29}$

\section{Postoperative}

Reduction in BMI following surgery can confound surgical outcomes. This is especially true in TORS, where dysphagia and odynophagia are inherent postoperative complications. Three studies revealed that the average BMI decreased by $0.5 \%$ from 29.0 to 28.8 after surgery. ${ }^{22,28,29} \mathrm{We}$ feel that this decrease in weight is not clinically significant in explaining the improvement in AHI, LSAT, and ESS. Cowan and Livingston reported that BMI will need to decrease by a minimum of $5 \mathrm{~kg} / \mathrm{m}^{2}$ in order to impact AHI. ${ }^{39}$ In addition, our pooled analysis revealed that the drop of -0.11 in BMI was not statistically significant $(95 \%$ CI, -0.75 to 0.54 ). Moreover, the dysphagia following TORS is often transient (discussed later). Therefore, a significant fall in BMI is purely on account of TORS is unlikely.

\section{Apnea-hypopnea index}

Lin et al stratified outcomes and reported that patients with preoperative AHI $<60$ had better surgical response rate of $67.9 \%$ in comparison to those with preoperative AHI $>60$ who had a response of only $18.2 \%{ }^{22}$ Kezirian et al in their evidence-based review on hypopharyngeal procedures combined two factors and reported that a BMI $>30$ and preoperative $\mathrm{AHI}>60$ corroborated with poorer outcomes in tongue tensioning procedures (genial tubercle advancement and hyoid suspension). ${ }^{16}$ Moreover, Caples et al reiterated the same fact that patients with lower BMI and AHI had the best outcomes in Phase I intervention. ${ }^{8}$ This would explain why RF-BOT would be recommended for patients with lower BMI with mild OSAHS but not for more severe OSAHS. ${ }^{40,41}$

\section{Site and type of collapse}

It is evident that in multilevel sleep surgery, precise identification of site of airway collapse is fundamental to achieve the best outcomes. In all studies, awake and sleep endoscopy were performed preoperatively. Chiffer et al additionally performed MRI preoperatively and postoperatively to evaluate how airway and soft tissue volumes changed following surgery. ${ }^{32}$ They found that changes in retropalatal and lateral wall soft tissue volume correlated significantly with the improvement in AHI. Lin et al reported that patients with lateral velopharyngeal collapse had a response rate of $25.0 \%$, whereas those without had a response rate of $66.7 \% .{ }^{22}$ This may prove to be useful during patient selection.

\section{Prior airway surgery}

Thaler et al compared outcomes between patients who had failed previous surgery with those who had never undergone surgery. ${ }^{23}$ They concluded that the operated group was challenging to treat with poor outcomes. It is imperative to recognize that despite our growing understanding of OSAHS, there remains a challenging group of patients who may fail regardless of all efforts. Future studies should be directed at evaluating this subgroup to improve outcomes and this may also prove worthwhile in preoperative counseling. Total sleep time $<90 \%$ oxygen saturation (hypoxic time) improved from $14 \%$ to $3.6 \%$ for patients who had not had previous OSAHS surgery and $21.1 \%$ to $12.5 \%$ in the group who had previous surgery. ${ }^{23}$

\section{Complications}

Complications resulting from TORS were minimal and classified as major if warranting surgical exploration and readmission and minor if managed conservatively.

\section{Tracheostomy}

The main concern in TORS is the risk of intraoperative or postoperative bleeding, which might be potentially difficult to arrest due to anatomical constraint of the vertical curvature of the BOT. This may pose a threat to the airway. In their preliminary study, Vicini et al routinely performed tracheostomy in their patients, but retrospectively concluded that this might not be necessary. ${ }^{18}$ Subsequently, some surgeons continued to perform tracheostomy. ${ }^{28,35}$ Others opted for less invasive measures such as overnight intubation, SICU, or HDU admission. ${ }^{22,29-31}$ However, it is reassuring to note that emergency tracheostomy was never required in any of the studies.

\section{Bleeding}

Bleeding from the laterally placed lingual artery can be potentially challenging to arrest. Vicini et al reported an intraoperative bleeding rate of $0.4 \% .{ }^{28}$ In this review, the volume of blood lost intraoperatively ranged from $12.9 \mathrm{~mL}^{22}$ to $27.7 \mathrm{~mL}^{21}$ with an average of $25.9 \mathrm{~mL} .^{21,22,33}$ To increase resection safety, Friedman et al employed the use of preoperative Doppler. ${ }^{26}$ However, this was likely required as they used the triangular resection technique, which was mucosal sparing but posed higher risk for the neurovasculature. Doppler may not be necessary if the traditional inverted pyramid technique is employed. ${ }^{27}$ 
We classified postoperative bleeding as major or minor depending on whether surgical exploration was undertaken. We found the average rate of major bleeding to be $2.9 \%$ and $0.5 \%$ for minor bleeding. In addition, bleeding did not occur from the BOT in the single case reported by Toh et al and six of seven cases reported by Glazer et al. ${ }^{29,31}$ Two case series noted that some cases of bleeding were associated with early restart of antithrombotics due to concomitant comorbidity. ${ }^{30,31}$ Therefore, the true rate of postoperative bleeding arising from the BOT is likely to be even lower. The morbidity is acceptable when compared to post-tonsillectomy hemorrhage rate of $4.8 \%$ reported by Bhattacharyya and Kepnes in a multicenter review of 7,748 adult tonsillectomies. ${ }^{42}$

\section{Pain and dehydration}

Given the anatomic proximity between neural structures and BOT, pain is expected and dehydration secondary to pain was not an uncommon complication postoperatively (Table 2). A common practice to complement analgesics and reduce airway edema was the perioperative usage of steroids as reported in many case series. ${ }^{22,26,27,29,33}$ Early toleration of oral feeding in the hospital setting may not necessarily translate to sufficient oral intake following discharge. Glazer et al reported a revisit rate of $10 \%$ due to dehydration. ${ }^{31}$ Hoff et al and Thaler et al reported that $5 \%$ of their patients required intravenous fluids. ${ }^{23,33}$ Thaler et al reported that none of their patients required readmission after they commenced gabapentin. ${ }^{23}$

Given the trend toward a shorter hospital stay of approximately 2 days, Glazer et al and Hoff et al proposed the implementation of home visits by a nurse following discharge, with patients with poor oral intake and severe pain being readmitted to a short-stay unit for treatment. ${ }^{31,33}$ Regardless of the time of discharge, patients should be given precise instructions to seek medical attention in the event of persistent pain and dehydration. Friedman et al mentioned that although patients undergoing robotic surgery stayed an average of 0.5 nights longer compared to patients undergoing RF-BOT, they did not experience more pain compared to the latter and patients undergoing SMILE on postoperative day $1 .{ }^{26}$

\section{Dysphagia}

Though swallowing is compromised after TORS, patients are able to gradually tolerate oral feeding on postoperative day 1 and diet of choice within 2 weeks after surgery. $28,29,35$ Certain studies opined that globus was related to the amount of energy used with cautery. ${ }^{26,31}$ In one case series, gastrostomy was needed and weaned off subsequently. ${ }^{31}$ Eesa et al administered the MD Anderson Dysphagia Inventory and found that there was no significant difference $(P=0.56)$ in self-reported swallowing function pre- and postoperatively. ${ }^{35}$ The authors also noted that gastrografin fluoroscopy at 1 week after surgery revealed the presence of aspiration in $24.4 \%$ of patients; however, this was not found to correlate to volume of tissue resection and resolved completely within 3 months without any remarkable postoperative weight loss. ${ }^{35}$ They suggested that subjective complaints of dysphagia may be perceived following surgery but these should not be subjected to unnecessary workup. ${ }^{35}$

\section{Dysgeusia}

The most common minor complication was dysgeusia, with a widely variable incidence of $14.2 \%-100 \% .^{28,35}$ Lin et al hypothesized that this may be caused by direct surgical injury to taste buds in the BOT and prolonged retraction, which results in stretching and neuropraxia of the lingual nerve. ${ }^{19}$ He recommended periodic interval relaxation to minimize this complication. Nevertheless, this complication is often transient in nature and resolves within 6 months to 1 year.

\section{Stenosis}

Three studies reported a rare complication of oropharyngeal stenosis in their patients (Table 2) ${ }^{22,28,34}$ This was managed by scar lysis or needle-point electrocautery and triamcinolone injection. Stenosis that can result from tonsillectomy can be potentially prevented by avoiding excessive cautery use and dissection in the region of inferior pole. ${ }^{43,44}$ These lessons may possibly be applicable to TORS as well.

\section{Others}

Other common postoperative complications included pharyngeal edema and tongue numbness. Vicini et al and Toh et al suggested that these may arise from the pressure from the tongue blade and that keeping the duration of time the patient is left in suspension to under 45 minutes may be useful. ${ }^{27,29}$

\section{TORS compared to other existing therapies for OSAHS}

\section{Other surgical procedures: tongue reduction and} tongue suspension

Comparing surgical outcomes between TORS and other hypopharyngeal surgeries is complicated, since preoperative $\mathrm{AHI}$ and BMI need to be matched to draw true conclusions. Needless to say if one were to factor in all the other PSG variables, it may render this task arduous, unattainable, and imprecise. 


\section{Tongue reduction}

Kezirian et al assessed the various hypopharyngeal surgical outcomes and reported unpredictable results with varied success ranging from as low as $20 \%$ to as high as $83 \% .{ }^{16}$ Another review focusing solely on the outcomes for glossectomy for OSAHS, which included other minimally invasive procedures and TORS, reported AHI reduction of $48.1 \pm 22.0$ to $19.1 \pm 15.5$ postoperatively. ${ }^{45}$ This is similar to our review finding, in which AHI dropped from 44.1 to 17.9 on average.

Friedman et al compared the outcomes of TORS with SMILE and RF-BOT. All three arms had the same palatal surgery, Z-palatopharyngoplasty. They reported that the robotic arm had significantly better outcomes in terms of AHI and surgical success when compared to the RF-BOT group. ${ }^{26}$ A plausible explanation is that the volume of tissue resected during TORS can be measured and is likely to be of a larger volume whereas tissue is vaporized during RF-BOT and cannot be quantified. Although RF-BOT is an outpatient procedure, it has lower efficacy compared to TORS and requires repeated treatments.

\section{Tongue tensioning procedures}

The surgical selection criterion to manage hypopharyngeal narrowing is dictated by the presence of micrognathia, retrusive chin, and/or BOT enlargement. Genial tubercle surgical advancements (mortised genioplasty and rectangular genial tubercle advancement procedures) and hyoid suspension (to the mandible or thyroid cartilage) alter the skeletal framework and work by altering the genioglossus muscle and upper airway muscles tension. In contrast, TORS primarily excises the excess lymphoid tissue with or without a small portion of muscle. Though the organ of manipulation is the same (BOT), it may not be fair to compare these procedures with TORS. There is a fundamental difference in dealing with skeletal framework and soft tissue resection. Regardless, skeletal framework surgery and TORS are complementary and not mutually exclusive.

\section{Others}

Maxillary mandibular advancement has superior results, addressing palatal and hypopharyngeal collapse. However, it has low acceptance and is reserved more as a salvage Phase II surgery. ${ }^{46}$

\section{Mortality improvement}

Surgery has been proven to reduce mortality in comparison to untreated controls. ${ }^{30}$ Weaver et al concluded that continuous positive airway pressure-treated veterans with OSAHS had a $31 \%$ higher probability of death compared to those treated by UPPP surgery. ${ }^{47}$ This may be due to inconsistent adherence or compliance to PAP therapy. In this context, multilevel surgery that yields better response than isolated palatal surgery can potentially lead to still better outcomes. Future studies should be directed toward this area.

\section{Limitations}

This review consists mainly of level 4 evidence in the form of predominantly retrospective case series without control arms. TORS was usually performed in conjunction with other surgical procedures and not in isolation. To ascertain the true efficacy of TORS in comparison with other existing BOT procedures, it may be worthwhile to analyze outcomes in isolated TORS. However, this may be impractical since severe OSAHS is a condition characterized by multilevel airway collapse.

\section{Adequacy of data}

Although eight of the 13 articles selected for review reported postoperative PSG outcomes, only results of the four studies comprising a sample of 329 patients were eventually used for statistical analysis. ${ }^{22,26,28,29}$ This stringent criteria were undertaken to minimize bias in the pooled results, as data published by some centers individually contributed toward data published by multicenter trials with both having overlapping recruitment periods. In such instances, the smaller studies were excluded from analysis. Prospective studies comprising larger sample size are required to truly ascertain the role of TORS in the multilevel surgical management of OSAHS. This may strengthen evidence-based decision-making.

\section{Reporting methods}

Another limitation was the inconsistent definition of surgical outcomes. Standardized reporting in sleep surgery is essential for evidence-based decision-making and comparisons. Even when surgical outcomes were clearly defined, results were not always presented clearly. One case series had well-defined stratified outcomes, presenting both proportional and cumulative rates. ${ }^{28}$ However, most reports needed to add more details on candidates who failed.

\section{Absence of postoperative PSG data}

Postoperative PSG data are essential to the assessment of outcomes and absence of this data may contribute toward bias. The total number of patients who underwent TORS exceeds the number who had postoperative PSG data. If this group of patients were tested with postoperative PSG study, the individual studies will have potentially higher sample size 
and greater information for analysis overall. Only one study cited reasons for absent postoperative PSG data that some patients declined due to financial concerns and improvement in snoring and daytime somnolence. ${ }^{29}$

\section{Evaluation of outcomes in sleep surgery}

All studies used the improvement in AHI to assess the effectiveness of surgery. Tam et al, however, stressed that outcomes in sleep surgery cannot be evaluated and confined to AHI reduction solely. ${ }^{48}$ Regrettably, none of the studies in our review included behavioral, sexual, functional, social, and quality-of-life outcomes systematically. A point to ponder is as surgeons, do we feel a sense of accomplishment if bed partners restart sleeping in the same room after snoring improves following surgery? Unfortunately, these indices are often not quantifiable.

\section{Follow-up duration}

The follow-up period of the included studies ranges from a minimum of 3 months to a maximum of 20 months, and most studies to 1 year. This length of follow-up may be inadequate, given that lingual lymphoid tissue may regrow over time, just as how adenoid tissue is capable of doing so. This may potentially result in disease recrudescence.

\section{Cure in OSAHS}

Cure is the intent in any treatment, but it may not be attainable in most chronic pathologies. ${ }^{49}$ Positive airway therapy ameliorates symptoms but does not cure OSAHS. Tracheostomy can surgically cure OSAHS but is not acceptable to most patients. Although the quest for the ideal minimally invasive surgery that yields predictable results in the management of OSAHS is still evolving, the need of the hour may be a relook into the assessment of outcomes.

Between the spectrum of cure and failure is a vast gray zone of potential improvement that deserves more credit and consideration in sleep surgery.

\section{Conclusion}

Technological advancement in the form of TORS offers safe resection of obstructing BOT and achieves improved AHI, ESS, and LSAT with acceptable morbidity. However, appropriate patient selection is critical to yield desirable results. Although the evidence suggests that TORS as a component of multilevel surgery in the treatment of OSAHS yields positive outcomes, insufficient data exist to evaluate its merit as a standalone procedure for tongue base reduction. Larger multicenter prospective randomized trials with longer duration of follow-up are required to evaluate its true clinical utility in the long term.

\section{Disclosure}

The authors report no conflicts of interest in this work.

\section{References}

1. Garvey JF, Pengo MF, Drakatos P, Kent BD. Epidemiological aspects of obstructive sleep apnea. J Thorac Dis. 2015;7(5):920-929.

2. Gami AS, Olson EJ, Shen WK, et al. Obstructive sleep apnea and the risk of sudden cardiac death: a longitudinal study of 10,701 adults. J Am Coll Cardiol. 2013;62(7):610-616.

3. Kendzerska T, Mollayeva T, Gershon AS, Leung RS, Hawker G, Tomlinson G. Untreated obstructive sleep apnea and the risk for serious long-term adverse outcomes: a systematic review. Sleep Med Rev. 2014;18(1):49-59.

4. Lee W, Nagubadi S, Kryger MH, Mokhlesi B. Epidemiology of obstructive sleep apnea: a population-based perspective. Expert Rev Respir Med. 2008;2(3):349-364.

5. Weaver TE, Grunstein RR. Adherence to continuous positive airway pressure therapy: the challenge to effective treatment. Proc Am Thorac Soc. 2008;5(2):173-178.

6. Campos-Rodriguez F, Peña-Griñan N, Reyes-Nuñez N, et al. Mortality in obstructive sleep apnea-hypopnea patients treated with positive airway pressure. Chest. 2005;128(2):624-633.

7. Aurora RN, Casey KR, Kristo D, et al. Practice parameters for the surgical modifications of the upper airway for obstructive sleep apnea in adults. Sleep. 2010;33(10):1408-1413.

8. Caples SM, Rowley JA, Prinsell JR, et al. Surgical modifications of the upper airway for obstructive sleep apnea in adults: a systematic review and meta-analysis. Sleep. 2010;33(10):1396-1407.

9. Lin HC, Friedman M, Chang HW, Gurpinar B. The efficacy of multilevel surgery of the upper airway in adults with obstructive sleep apnea/ hypopnea syndrome. Laryngoscope. 2008;118(5):902-908.

10. Ravesloot MJ, de Vries N. One hundred consecutive patients undergoing drug-induced sleep endoscopy: results and evaluation. Laryngoscope. 2011;121(12):2710-2716.

11. Vroegop AV, Vanderveken OM, Boudewyns AN, et al. Drug-induced sleep endoscopy in sleep-disordered breathing: report on 1,249 cases. Laryngoscope. 2014;124(3):797-802.

12. Friedman M, Wilson MN, Pulver TM, et al. Measurements of adult lingual tonsil tissue in health and disease. Otolaryngol Head Neck Surg. 2010;142(4):520-525.

13. Saboisky JP, Stashuk DW, Hamilton-Wright A, et al. Neurogenic changes in the upper airway of patients with obstructive sleep apnea. Am J Respir Crit Care Med. 2012;185(3):322-329.

14. Nashi N, Kang S, Barkdull GC, Lucas J, Davidson TM. Lingual fat at autopsy. Laryngoscope. 2007;117(8):1467-1473.

15. Robinson S. External submucosal glossectomy. In: Friedman M, editor. Sleep Apnea and Snoring: Surgical and Non-Surgical Therapy. New York: Elsevier; 2008:292-300.

16. Kezirian EJ, Goldberg AN. Hypopharyngeal surgery in obstructive sleep apnea: an evidence-based medicine review. Arch Otolaryngol Head Neck Surg. 2006;132(2):206-213.

17. O’Malley BW Jr, Weinstein GS, Snyder W, Hockstein NG. Transoral robotic surgery (TORS) for base of tongue neoplasms. Laryngoscope. 2006;116(8):1465-1472.

18. Vicini C, Dallan I, Canzi P, Frassineti S, La Pietra MG, Montevecchi F. Transoral robotic tongue base resection in obstructive sleep apnoeahypopnoea syndrome: a preliminary report. ORL J Otorhinolaryngol Relat Spec. 2010;72(1):22-27.

19. Lin HS, Rowley JA, Badr MS, et al. Transoral robotic surgery for treatment of obstructive sleep apnea-hypopnea syndrome. Laryngoscope. 2013;123(7):1811-1816. 
20. Lee JM, Weinstein GS, O'Malley BW, Jr, Thaler ER. Transoral robotassisted lingual tonsillectomy and uvulopalatopharyngoplasty for obstructive sleep apnea. Ann Otol Rhinol Laryngol. 2012;121(10): 635-639.

21. Vicini C, Dallan I, Canzi P, et al. Transoral robotic surgery of the tongue base in obstructive sleep apnea-hypopnea syndrome: anatomic considerations and clinical experience. Head Neck. 2012;34(1):15-22.

22. Lin HS, Rowley JA, Folbe AJ, Yoo GH, Badr MS, Chen W. Transoral robotic surgery for treatment of obstructive sleep apnea: factors predicting surgical response. Laryngoscope. 2015;125(4):1013-1020.

23. Thaler ER, Rassekh CH, Lee JM, Weinstein GS, O'Malley BW, Jr. Outcomes for multilevel surgery for sleep apnea: obstructive sleep apnea, transoral robotic surgery, and uvulopalatopharyngoplasty. Laryngoscope. 2016;126(1):266-269.

24. Muderris T, Sevil E, Bercin S, Gul F, Kiris M. Transoral robotic lingual tonsillectomy in adults: preliminary results. Acta Otolaryngol. 2015;135(1):64-69.

25. Nice.org.uk [homepage on the Internet]. NICE Quality Assessment for Case Series. Available from: http://www.nice.org.uk/guidance/cg3/resources/ appendix-4-quality-of-case-series-form2. Accessed June 12, 2015.

26. Friedman M, Hamilton C, Samuelson CG, et al. Transoral robotic glossectomy for the treatment of obstructive sleep apnea-hypopnea syndrome. Otolaryngol Head Neck Surg. 2012;146(5):854-862.

27. Vicini C, Montevecchi F, Magnuson JS. Robotic surgery for obstructive sleep apnea. Curr Otorhinolaryngol Rep. 2013;1:130-136.

28. Vicini C, Montevecchi F, Campanini A, et al. Clinical outcomes and complications associated with TORS for OSAHS: a benchmark for evaluating an emerging surgical technology in a targeted application for benign disease. ORL J Otorhinolaryngol Relat Spec. 2014;76(2):63-69.

29. Toh ST, Han HJ, Tay HN, Kiong KL. Transoral robotic surgery for obstructive sleep apnea in Asian patients: a Singapore sleep centre experience. JAMA Otolaryngol Head Neck Surg. 2014;140(7):624-629.

30. Hoff PT, Glazer TA, Spector ME. Body mass index predicts success in patients undergoing transoral robotic surgery for obstructive sleep apnea. ORL J Otorhinolaryngol Relat Spec. 2014;76(5):266-272.

31. Glazer TA, Hoff PT, Spector ME. Transoral robotic surgery for obstructive sleep apnea: perioperative management and postoperative complications. JAMA Otolaryngol Head Neck Surg. 2014;140(12):1207-1212.

32. Chiffer RC, Schwab RJ, Keenan BT, Borek RC, Thaler ER. Volumetric MRI analysis pre- and post-transoral robotic surgery for obstructive sleep apnea. Laryngoscope. 2015;125(8):1988-1995.

33. Hoff PT, D'Agostino MA, Thaler ER. Transoral robotic surgery in benign diseases including obstructive sleep apnea: safety and feasibility. Laryngoscope. 2015;125(5):1249-1253.

34. Muderris T, Sevil E, Bercin S, Gul F, Kiris M. Oropharyngeal stenosis after transoral robotic lingual tonsillectomy. J Craniofac Surg. 2015;26(3):853-855.
35. Eesa M, Montevecchi F, Hendawy E, D'Agostino G, Meccariello G, Vicini C. Swallowing outcome after TORS for sleep apnea: shortand long-term evaluation. Eur Arch Otorhinolaryngol. 2015;272(6): $1537-1541$.

36. Do KL, Ferreyra H, Healy JF, Davidson TM. Does tongue size differ between patients with and without sleep-disordered breathing? Laryngoscope. 2000;110(9):1552-1555.

37. Schwartz AR, Patil SP, Squier S, Schneider H, Kirkness JP, Smith PL. Obesity and upper airway control during sleep. J Appl Physiol (1985). 2010;108(2):430-435.

38. Li KK, Kushida C, Powell NB, Riley RW, Guilleminault C. Obstructive sleep apnea syndrome: a comparison between Far-East Asian and white men. Laryngoscope. 2000;110(10 Pt 1):1689-1693.

39. Cowan DC, Livingston E. Obstructive sleep apnoea syndrome and weight loss: review. Sleep Disord. 2012;2012:163-296.

40. Fernández-Julián E, Muñoz N, Achiques MT, García-Pérez MA, Orts M, Marco J. Randomized study comparing two tongue base surgeries for moderate to severe obstructive sleep apnea syndrome. Otolaryngol Head Neck Surg. 2009;140(6):917-923.

41. Riley RW, Powell NB, Li KK, Weaver EM, Guilleminault C. An adjunctive method of radiofrequency volumetric tissue reduction of the tongue for OSAS. Otolaryngol Head Neck Surg. 2003;129(1):37-42.

42. Bhattacharyya N, Kepnes LJ. Revisits and postoperative hemorrhage after adult tonsillectomy. Laryngoscope. 2014;124(7):1554-1556.

43. Santos VB, Ruffy ML, Polisar IA. Stenosis of the oropharynx treated with intralesional triamcinolone. Ear Nose Throat J. 1977;56(4):164-167.

44. McLaughlin KE, Jacobs IN, Todd NW, Gussack GS, Carlson G. Management of nasopharyngeal and oropharyngeal stenosis in children. Laryngoscope. 1997;107(10):1322-1331.

45. Murphey AW, Kandl JA, Nguyen SA, Weber AC, Gillespie MB. The effect of glossectomy for obstructive sleep apnea: a systematic review and meta-analysis. Otolaryngol Head Neck Surg. 2015;153(3):334-342.

46. Riley RW, Powell NB, Guilleminault C. Obstructive sleep apnea syndrome: a surgical protocol for dynamic upper airway reconstruction. J Oral Maxillofac Surg. 1993;51(7):742-747; discussion 748-749.

47. Weaver EM, Maynard C, Yueh B. Survival of veterans with sleep apnea: continuous positive airway pressure versus surgery. Otolaryngol Head Neck Surg. 2004;130(6):659-665. Erratum in: Otolaryngol Head Neck Surg. 2004;131(1):144.

48. Tam S, Woodson BT, Rotenberg B. Outcome measurements in obstructive sleep apnea: beyond the apnea-hypopnea index. Laryngoscope. 2014;124(1):337-343.

49. Weaver EM. Judging sleep apnea surgery. Sleep Med Rev. 2010;14(5): 283-285.
Robotic Surgery: Research and Reviews

\section{Publish your work in this journal}

Robotic Surgery: Research and Reviews is an international, peer reviewed, open access, online journal publishing original research, commentaries, reports, and reviews on the theory, use and application of robotics in surgical interventions. Articles on the use of supervisorycontrolled robotic systems, telesurgical devices, and shared-control
Dovepress

systems are invited. The manuscript management system is completely online and includes a very quick and fair peer review system, which is all easy to use. Visit http://www.dovepress.com/testimonials.php to read real quotes from published authors. 